TAO, Vol. 17, No. 1, 253-261, March 2006

\title{
The Determination of Sunglint Locations on the Ocean Surface by Observation from Geostationary Satellites
}

\author{
E. G. Emecen ${ }^{1}$, G. Kara ${ }^{1, *}$, F. Erdoğmuş ${ }^{1}$ and R. Gardashov ${ }^{1}$
}

(Manuscript received 6 July 2004, in final form 10 November 2005)

\begin{abstract}
The method of determining a principal point of sunglint (PPS) on the ocean surface by observation from geostationary orbit is described. To find a PPS first a nonlinear equation is solved numerically, and then from one transformation the coordinates (latitude and longitude) of the PPS are obtained. The diurnal, seasonal and annual excursion of PPS are investigated. The entry parameters of the problem are the time $t$ and longitude of satellite $\varphi_{\text {sat }}$. The contour of the Sun disk image and its sizes on a smooth ocean surface are studied. This method of finding PPS is then tested upon the Earth's image recorded from Meteosat satellites.
\end{abstract}

(Key words: Geostationary satellites, Sea surface, Sunglint, Reflection)

\section{INTRODUCTION}

The study of the characteristics of light reflected from the sea surface is important in optical remote sensing of physical and geometrical properties of the sea surface. For example, average intensity of reflected sunlight at different angles of incidence and view gives the statistical distribution of surface slopes (Cox and Munk 1954). Thus, when properly calculated and understood, the reflection of sunlight from the ocean surface, termed sunglint, can provide useful information about the nature of the reflecting surface as well as processes occurring nearby. In addition, this information is needed for careful selection of satellite orbit and sensor viewing geometry in measurements, which consider solar reflection as a nuisance. For in-

\footnotetext{
${ }^{1}$ Marine Transportation and Management Department, Engineering Faculty, University of Istanbul, Istanbul, Turkey

* Corresponding author address: Dr. Gökhan Kara, Marine Transportation and Management Department, University of Istanbul, Engineering Faculty , Avcilar, Istanbul, Turkey; E-mail: karagok@istanbul.edu.tr
} 
stance in the paper Prakash et al. (1994) considered the problem of precise location of the PPS that is determined by solving a system of non-linear equations in two variables using the Newton-Raphson method, which includes difficulties connected with convergence.

The aim of this present work is to develop a comparatively simple method by which the PPS can be determined by solving a nonlinear equation, opposite to the system of equations in paper Prakash et al. (1994). This simplification has been reached by choosing a suitable reference plane, that is, the plane passing through the Sun's center, Earth's center and satellite.

Earth images recorded for a certain time from the geostationary orbit at the Meteosat satellite are disseminated by the European Organization for the Exploitation of Meteorological Satellites (EUMETSAT). The location of the PPS on these images depends on time $t$ (which is measured in terms of GMT) and on position of satellite, determined by angle $\varphi_{\text {sat }}$ of satellite longitude. So far as the parameters $t$ and $\varphi_{\text {sat }}$ are known for any images, a method which determines the PPS precisely for a given $t$ and $\varphi_{\text {sat }}$, may be developed.

\section{THE GEOMETRY AND BASIC RELATIONSHIP OF THE PROBLEM}

The Earth, which is assumed to be an oblate spheroid, makes orbital motion around the Sun and geostationary satellites produce observation of the Earth. The geometry of the problem is represented in Fig.1, where ADLPB is an arc of the equator; ASCB is an arc of section of the orbital plane and Earth. The point $O$ is the center of the Earth; the point $Q$ is the position

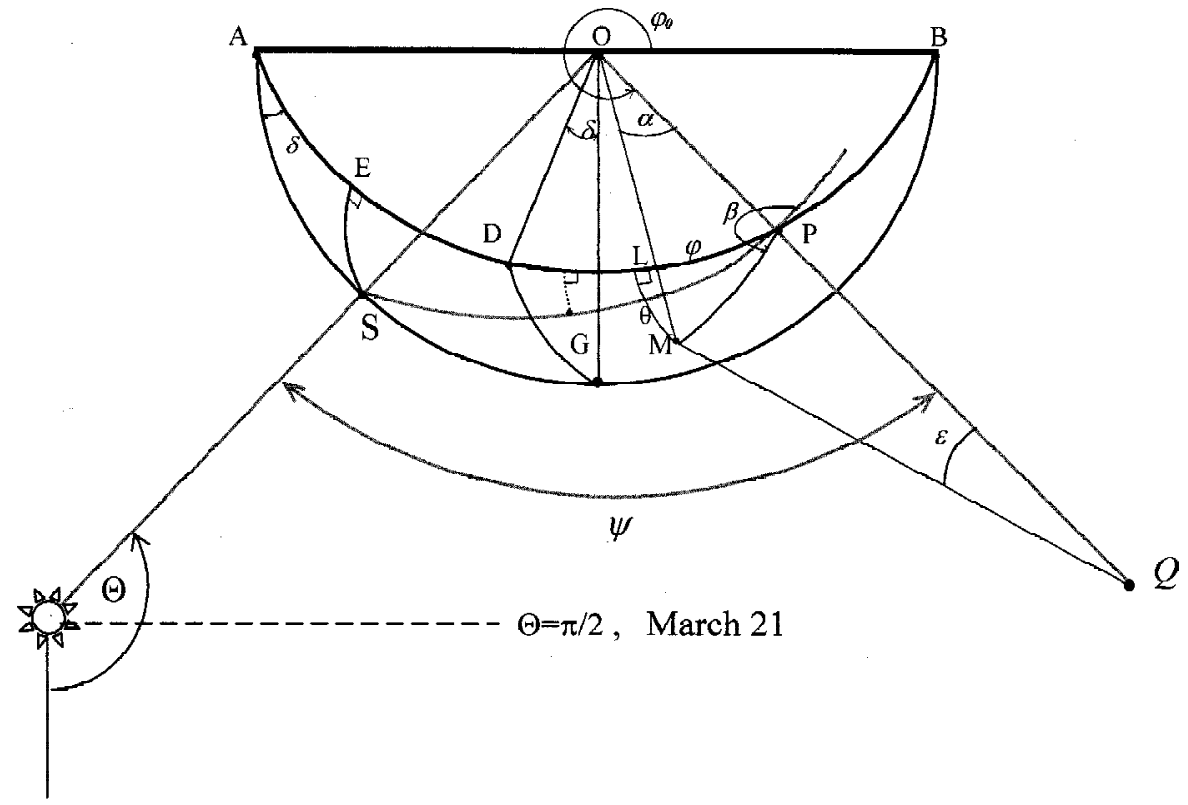

Fig. 1. The geometry of the problem. 
of the geostationary satellite which is determined by the longitude $\varphi_{\text {sat }}$. The arbitrary point $M$ on the Earth can be defined by angles $\alpha$ (or $\varepsilon$ ) and $\beta$ (angle $\beta$ is measured from the reference plane SOP). The same point $M$ can also be defined by angles of latitude, $\theta$, and longitude $\varphi$. The angle $\varphi=\operatorname{arc}(P L)$, i.e., it is measured from the direction to satellite and therefore differs from a real longitude of $M$ by the angle $\varphi_{\text {sat }}$. PPS is a point $G$ on the arc SP, which reflects the ray traveling from the centre of the Sun to the point of observation $Q$.

In the first step, the position of the Earth is determined by solving of Kepler's equations of the Earth's orbital motion:

$$
M=E-e \sin E,
$$

where, $E$ is the eccentric anomaly, $M$ is the mean anomaly. The eccentric anomaly $E$ is connected with true anomaly, $\Theta$ by equation:

$$
\Theta=2 \arctan \left(\sqrt{\frac{1+e}{1-e}} \tan \frac{E}{2}\right),
$$

where, $e=0.016729$ is the eccentricity of the Earth's orbit. The mean anomaly relates to time $t(s)$ as,

$$
M=\frac{\sqrt{G M_{s}}}{a^{3 / 2}} t \approx 0.199 \times 10^{-6} \cdot t
$$

where, $M_{s}=1.989 \times 10^{30} \mathrm{~kg}$ is the Sun mass, $G=6.672 \times 10^{11} \mathrm{~N} \mathrm{~m}^{2} \mathrm{~kg}^{-2}$ is the gravitational constant, $a=1.496 \times 10^{8} \mathrm{~km}$ semimajor axis of the elliptical Earth orbit. The solution (1) and (2) gives dependence $\Theta=\Theta(t)$, i.e., the position of the Earth on the orbit (see Fig. 1). There are different numerical methods for solving Kepler's equations (Taff 1985).

In the second step, at any time $t$ the position of the satellite is determined. This means that the angle:

$$
\varphi_{Q}=\varphi_{\text {sat }}+\omega t
$$

$(\omega=2 \pi / T, T=23.96$ hours $=23.96 \times 3600 \mathrm{sec}$. is the sidereal period of Earth's rotation $)$ is defined. The time $t(s)$ is reckoned from time instant: GMT $=0.00,21$ March. The angle $\psi=\angle S O Q$ is found from:

$$
\cos \psi=-\sin \theta \cos \varphi+\cos \theta \sin \varphi \cos \delta
$$

where $\delta=23^{\circ} 27^{\prime}$ is the angle of inclination (declination). The angle $\alpha=\alpha_{G}$, which determine the PPS (the point G in Fig. 1), is found as a root of equation: 


$$
2 \alpha+\varepsilon(\alpha)-\psi=0,
$$

and the angle $\beta$ of point $\mathrm{G}$ is $\beta_{G}=\pi$. The function $\varepsilon=\varepsilon(\alpha)$ is given by:

$$
\varepsilon=\varepsilon(\alpha)=\arctan \left(\frac{\tau \sin \alpha}{1-\tau \cos \alpha}\right),
$$

where $\tau \approx 0.1513$. A behavior of $\varepsilon=\varepsilon(\alpha)$ was analyzed in (Gardashov and Barla 2001), where it has been shown that the intervals in which $\alpha$ and $\varepsilon$ changes is: $0^{\circ} \leq \alpha \leq 81.30^{\circ}$ and $0^{\circ} \leq \varepsilon$ $\leq 8.70^{\circ}$. Note, that locating the solar image is very similar to the problem of locating the point of specular reflection from the Earth, which is solved in the radio science publications for a more general case (Martin-Neira 1993).

There is no difficulty solving equation (5) using numerical methods. Because, it's easy to separate the interval in which the root is isolated and no problem of convergence of iteration arises. Therefore, using the numerical methods the root of equation (5) can be obtained with the needed tolerance.

The transformation from angles $(\alpha, \beta)$ to angles of latitude $\theta$ and longitude $\varphi$ of point $M$ can be performed using the formulas of spherical geometry:

$$
\left.\begin{array}{l}
\sin \theta=\sin \alpha \cdot \sin (\beta-\pi+P) \\
\sin \varphi=\tan \theta \cdot \cot (\beta-\pi+P)
\end{array}\right\},
$$

where $P=\angle S P A$. is determined by $P=\arcsin (\cos \varphi \cdot \sin \delta / \sin \psi)$. For the PPS (i.e., when $M \equiv G) \alpha=\alpha_{G}$ and $\beta=\beta_{G}=\pi$ and from (6) we find angles $\theta_{G}$ and $\varphi_{G}$ which are the position, i.e.,latitude and longitude, of PPS.

Computing angles $\theta_{G}, \varphi_{G}$ for different moments of time $t$ described by the above method the relationships $\theta_{G}=\theta_{G}(t), \varphi_{\mathrm{G}}=\varphi_{\mathrm{G}}(t)$ are thus obtained, which in appropriate intervals of changes of $t$ gives diurnal, seasonal and annual excursion of PPS.

\section{APPLICATION OF THE METHOD}

The result of calculating this method for satellite "Meteosat" located at latitude $\varphi_{\text {sat }}=63^{\circ}$ East is shown in Fig. 2. The thick lines for different months are the predicted diurnal path of PPS as seen from the satellite. The annual excursion of PPS for the particular time of the day is given by a closed curve. The seasonal excursion is included year to year and in Fig. 2 is shown by star signs.

The asymmetric behavior of closed curves describing the annual excursion of PPS is connected with the elliptical form of the Earth orbit (if formally takes the orbit as a cycle i.e., the eccentricity $e=0$ then the curves becomes a symmetric form). In Fig. 3, the image of the 


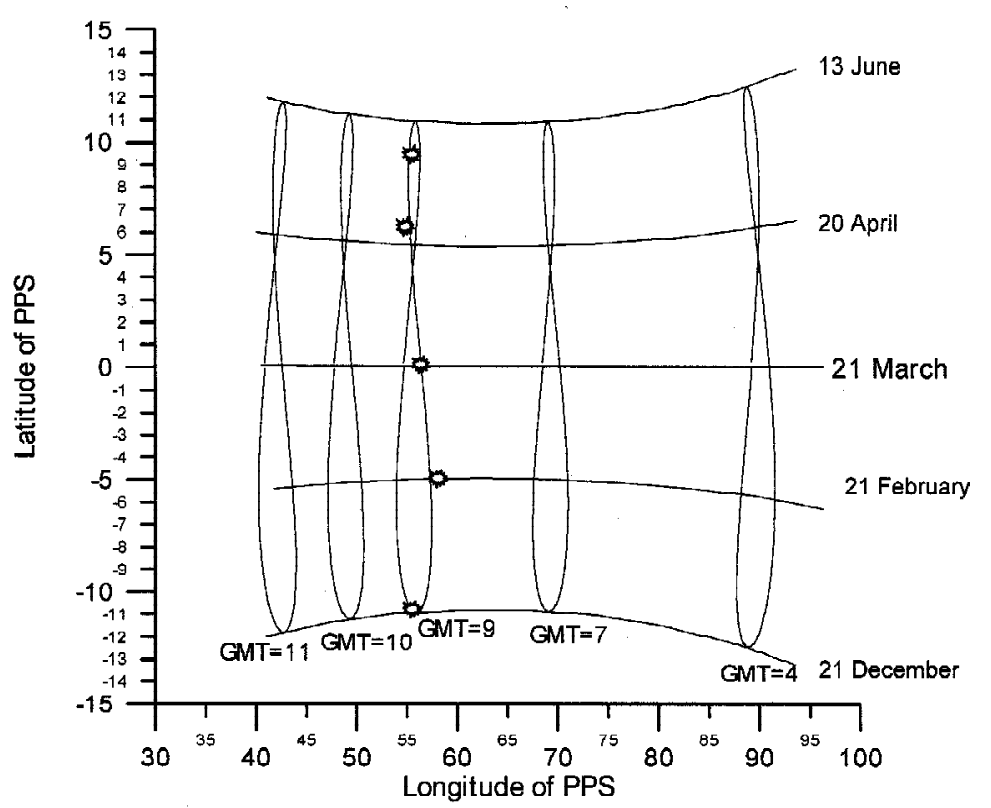

Fig. 2. The annual excursion of PPS (closed curves) for the times GMT=4,7,9, 10,11 and diurnal excursion (from East to West) and seasonal (from North to South).

Earth obtained from this satellite on 20 April, GMT $=09: 00$ is represented. The region occupied by sunglint is clearly viewed. The coordinates for the center of this region are: $\theta \approx 5^{\circ}$, $\varphi \approx 54^{\circ}$ which coincide with the coordinates of PPS, $\theta_{G}, \varphi_{G}$ calculated by our method. In Fig. 2, PPS is shown by a star. The meridians and parallels on the image are drawn by using FORTRAN programmer from EUMETSAT (The Meteosat Archive User Handbook 2001), which produces transformation from numerical (line/pixel) coordinates of an image to geographical (lat. /long.) coordinates.

The series of images in which sunglint appears clearly were analyzed by us. Some images of when the sunglint area is viewed are represented in Table 1.

As seen in Table 1, for all of these images the centers of occupied sunglint are located in about the same locations for PPS calculated by the method described above for corresponding time $t$ and longitude of satellite $\varphi_{\text {sat }}$.

\section{THE CONTOUR OF THE SUN DISK IMAGE}

Now suppose that the ocean's surface is absolutely smooth and let us define the location of the sunglint (i.e., Sun disk image on the ocean surface). Because the angular size of the Sun is very small, but not zero, the images of the Sun on the ocean will also have finite sizes. The 


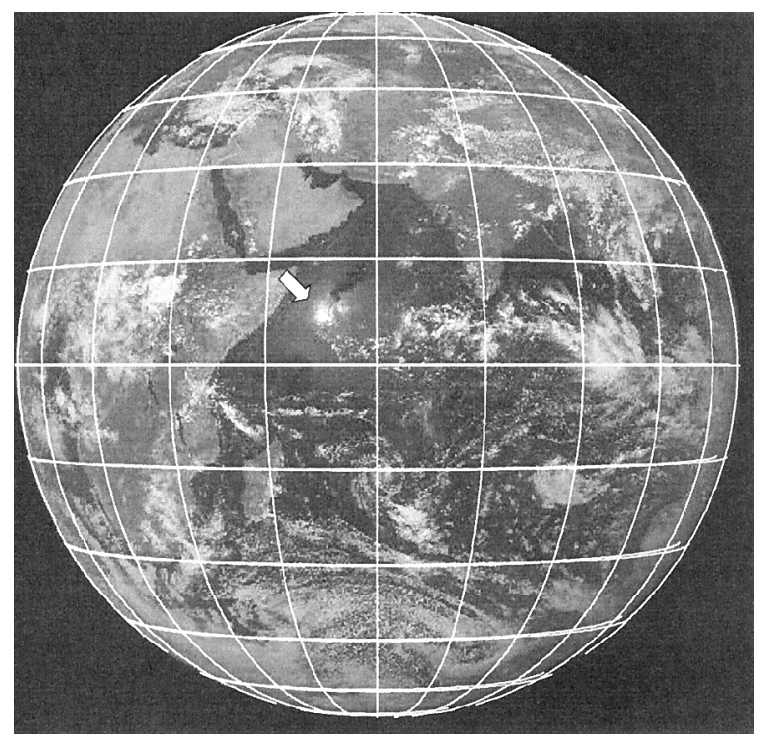

Fig. 3. The Earth image recorded from Meteosat with parallel and meridian lines designed using EUMETSAT program (21 April 2000, GMT: 09:00).

Table 1. PPS coordinates derived from images and calculations.

\begin{tabular}{|c|l|l|}
\hline \multicolumn{1}{|c|}{ Satellite Image } & $\begin{array}{c}\text { Latitude-Longitude } \\
\text { derived from images }\end{array}$ & $\begin{array}{c}\text { Latitude-Longitude } \\
\text { calculated }\end{array}$ \\
\hline 13 JUNE GMT $=09: 00$ & $\theta \approx 11^{0}, \varphi \approx 54^{0}$ & $\theta=10,9^{\circ}, \varphi=54,0^{\circ}$ \\
\hline 20 APRIL GMT $=09: 00$ & $\theta \approx 5^{0}, \varphi \approx 54^{0}$ & $\theta=05,3^{0}, \varphi=53,8^{0}$ \\
\hline 21 MARCH GMT $=09: 00$ & $\theta \approx 0^{0}, \varphi \approx 55^{0}$ & $\theta=0,1^{0}, \varphi=54,7^{0}$ \\
\hline 21 FEBRUARY GMT $=09: 00$ & $\theta \approx-1^{0}, \varphi \approx 57^{0}$ & $\theta=-05,0^{\circ}, \varphi=55,6^{\circ}$ \\
\hline 21 DECEMBER GMT $=09: 00$ & $\theta \approx-11^{0}, \varphi \approx 54^{0}$ & $\theta=-11,0^{0}, \varphi=54,2^{0}$ \\
\hline
\end{tabular}

angles $(\alpha, \beta)$ of point $M$ at the contour of the Sun disk images satisfy the following equation (Gardashov and Barla 2001; Kara et al. 2001).

$$
\begin{aligned}
& \cos \left(\beta-\beta_{G}\right)\left[\sin \varepsilon \sin \varepsilon_{G}+2 \sin \varepsilon \sin \alpha_{G} \cos \bar{\theta}_{G}+\right. \\
& \left.2 \sin \alpha_{G} \sin \varepsilon \cos \bar{\theta}+4 \sin \alpha \sin \alpha_{G} \cos \bar{\theta} \cos \bar{\theta}_{G}\right]+ \\
& \cos \varepsilon_{G} \cos \varepsilon_{G}-2 \cos \varepsilon \cos \alpha_{G} \cos \bar{\theta}_{G}- \\
& 2 \cos \varepsilon_{G} \cos \alpha \cos \bar{\theta}+4 \cos \alpha \cos \alpha_{G} \cos \bar{\theta} \cos \bar{\theta}_{G}=\cos \frac{\gamma_{s}}{2},
\end{aligned}
$$


where $\gamma_{s}=d_{s} / R_{s}=0.54^{\circ}$ linear angle of the Sun disk (diameter $d_{s}=1.3914 \times 10^{6} \mathrm{~km}$ the diameter of Sun, $R_{s}=1.49 \times 10^{8} \mathrm{~km}$ the mean distance between the Earth and the Sun), $\varepsilon=\varepsilon(\alpha)$, $\varepsilon_{G}=\varepsilon\left(\alpha_{G}\right)$ and $\bar{\theta}=\alpha+\varepsilon(\alpha), \bar{\theta}_{G}=\alpha_{G}+\varepsilon\left(\alpha_{G}\right)$. Where, $\alpha_{G}$ corresponds to the ray going to $\mathrm{Q}$ from the Sun disk center after reflection. Equation (8) in implicate form defines a function $\alpha=\alpha_{b}(\beta)$, which describes the contour of the Sun disk image on the surface. Solving numerically equation (8), i.e., finding values $\alpha$ for each values $\beta$ from a certain interval of changes in $\beta$, we can draw a graphic of the function $\alpha=\alpha_{b}(\beta)$. The graphs of the function $\alpha=\alpha_{b}(\beta)$, i.e., the contour of the Sun disk image, at the angles $\alpha_{G}=45^{\circ}, 60^{\circ}$ and $72^{\circ}$ are shown in Fig. 4. The corresponding values of angle $\psi_{G}$, findings from the equation (5) are: $\psi_{G}=96.83^{\circ}, 128.07^{\circ}$ and $152.58^{\circ}$.

As can be seen the Sun disk image forms look elliptical. The Sun disk image diameters $l_{\alpha}$ and $l_{\beta}$ along the angles $\alpha$ and $\beta$ can be estimated as follows (Gardashov and Barla 2001):

$$
\psi=2 \alpha+\varepsilon(\alpha) \quad, \quad \Delta \psi=2 \Delta \alpha+\varepsilon^{\prime}(\alpha) \Delta \alpha .
$$

If we take $\Delta \psi=\gamma_{s}$ (where $\gamma_{s}=d_{s} / R_{s}=0.54^{\circ}, d_{s}=1.3914 \times 10^{6} \mathrm{~km}$ diameter of the Sun, $R_{s}=1.49 \times 10^{8} \mathrm{~km}$ mean distance between the Sun and the Earth) and $\alpha=\alpha_{c}$ we have:

$$
\Delta \alpha=\frac{\gamma_{s}}{2+\varepsilon^{\prime}\left(\alpha_{G}\right)}, \quad l_{\alpha}=R \cdot \Delta \alpha=\frac{R \gamma_{s}}{2+\varepsilon^{\prime}\left(\alpha_{G}\right)} .
$$

We can also find that (where, $R=6378 \mathrm{~km}$ is Earth radius)

$$
\sin \frac{\Delta \beta}{2}=\frac{\gamma_{s}}{2 \sin \psi_{G}} \Delta \beta=2 \sin ^{-1}\left(\frac{\gamma_{s}}{2 \sin \psi_{G}}\right) \text { and } t_{\beta}=\frac{R \sin \alpha_{G}}{\sin \psi_{G}} \gamma_{s},
$$

as $\alpha_{G} \rightarrow 0$ we have:

$$
\frac{\sin \alpha_{G}}{\sin \psi_{G}} \approx \frac{\alpha_{G}}{\psi_{G}}=\frac{\alpha_{G}}{2 \alpha_{G}+\varepsilon\left(\alpha_{G}\right)} \approx \frac{\alpha_{G}}{2 \alpha_{G}+\varepsilon^{\prime}(0) \alpha_{G}}=\frac{1}{2+\varepsilon^{\prime}(0)},
$$

and we see that $l_{\alpha}=l_{\beta}$ at $\alpha_{G}=0$.

The values of the Sun disk image diameters $l_{\alpha}$ and $l_{\beta}$ along $\alpha$ and $\beta$ for different $\alpha_{G}\left(\right.$ or $\psi_{G}$ ) are given in the Table 2.

\section{CONCLUSION}

When waves appear on the ocean surface the whole Sun disk image is divided into glints. As the height of the surface waves increase the area that sun glitters occupy widens. The sizes 

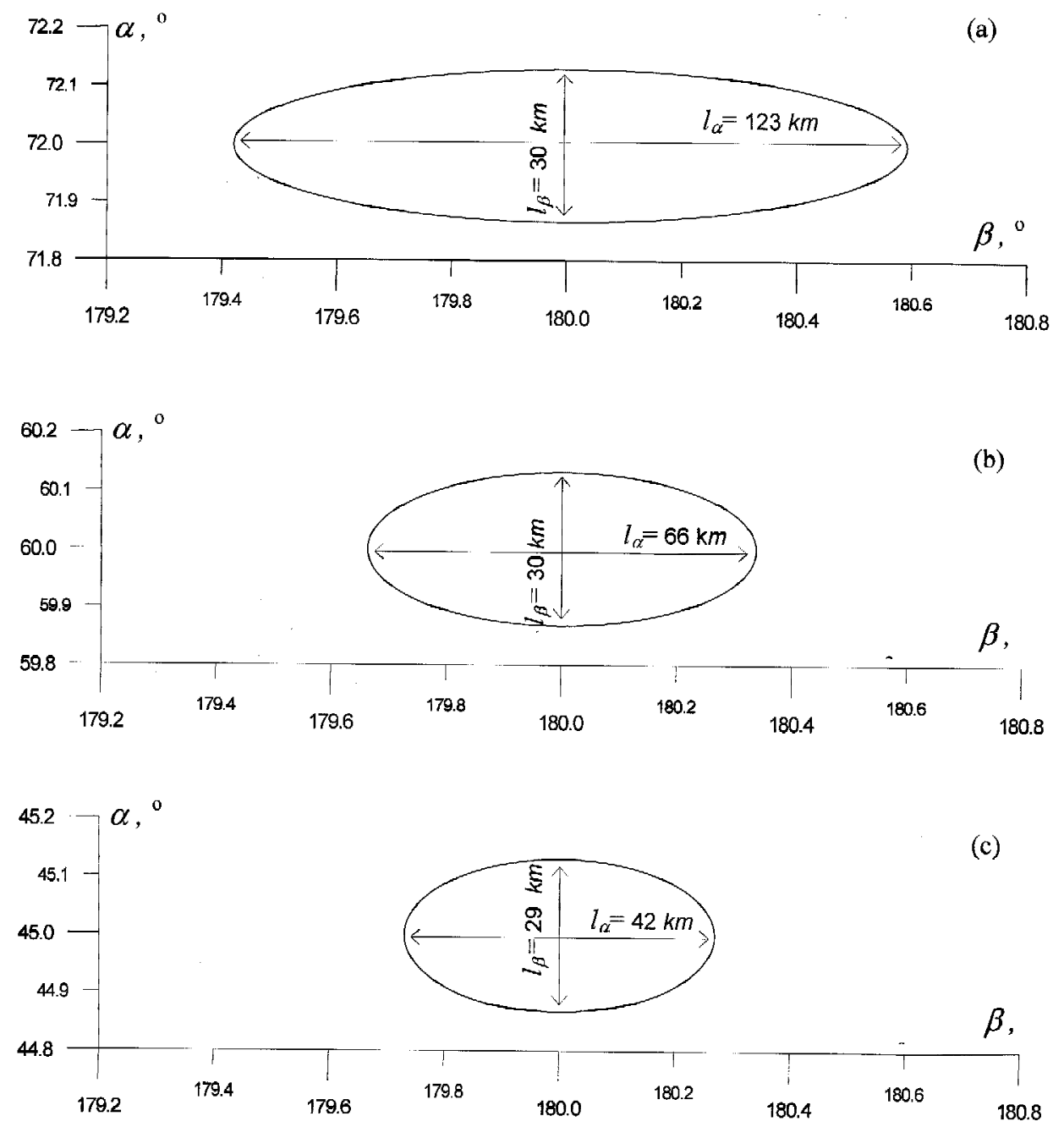

Fig. 4. The forms and sizes of the glint at the angle $\psi_{G}$ : (a) $\psi_{G}=96.83^{\circ}$, $\left(\alpha_{G}=45^{\circ}\right)$; (b) $\psi_{G}=128.07^{\circ},\left(\alpha_{G}=60^{\circ}\right)$; (c) $\psi_{G}=152.58^{\circ},\left(\alpha_{G}=72^{\circ}\right)$.

Table 2. The values of linear sizes $l_{\alpha}$ and $l_{\beta}$ of the Sun disk image.

\begin{tabular}{|l|c|c|c|c|c|c|c|c|}
\hline$\left.\alpha_{G},{ }^{\circ}\right)$ & $\mathbf{0}$ & $\mathbf{3 0}$ & $\mathbf{4 5}$ & $\mathbf{6 0}$ & $\mathbf{7 2}$ & $\mathbf{7 5}$ & $\mathbf{8 0}$ & $\mathbf{8 1 . 3 0}$ \\
\hline$\left.\psi_{G},{ }^{\circ}\right)$ & 0 & 64.98 & 96.83 & 128.07 & 152.58 & 158.65 & 168.70 & 171.30 \\
\hline$l_{\alpha}, \mathrm{km}$ & 27.3 & 27.8 & 28.3 & 28.9 & 29.4 & 29.5 & 29.7 & 29.8 \\
\hline$l_{\beta}, \mathrm{km}$ & 27.3 & 32.9 & 42.4 & 65.5 & 123.0 & 158.0 & 299.3 & 389.3 \\
\hline
\end{tabular}


of this region and distribution of glitter brightness in this region depend on wind and wave fields. Consequently, from the sunglint images of the ocean surface these field parameters can be derived. The accuracy of this method will basically be determined by the accuracy of atmospheric influence and surface slope distribution functions. Supposing that ocean wave characteristics in the observation region of sunglint are homogeneous, the calculation of wave and wind parameters can be produced (Prakash et al. 1994; Khattak et al. 1991). In this case accuracy may be estimated by comparison with the results of direct experiments.

\section{REFERENCES}

Cox, C., and W. Munk, 1954: Measurement of the roughness of the sea surface from Sun's glitter. J. Opt. Soc. Am., 44, 834-850.

Gardashov, R., and M. C., Barla, 2001: The calculation of the distribution of the Sunglint radiance on the ocean surface by observing from a geostationary orbit. Int. J. Remote Sens., 22, 2939-2952.

Kara, G., M. C. Barla, F. Erdoğmuş, E. G. Emecen, and R. Gardashov, 2001: Method To Locate Sunglint On The Ocean Surface By Observing From The Geostationary Orbit, Current Problems in Optics of Natural Waters, ONW, I. Int. Conf., 25-29 Sept., SaintPetersburg, Russia, 152-156.

Khattak, S., R. A.Vaughan, and A. P. Cracknell, 1991: Sunglint and its observation in AVHRR data. Remote Sens. Environ., 37, 101-116.

Martin-Neira, M., 1993: A Passive Reflectometry and Interferometry System (PARIS): application to ocean altimetry. ESA J., 17, 331-355.

Prakash, W. J., A. K. Varma, and S. M. Bhandari, 1994: An algorithm for the precise location of the solar specular reflection point in the visible band images from geostationary meteorological satellites. Comp. Geosci., 20, 1467-1482.

Taff, L. G.,1985: Celestial Mechanics. Chichester, John Wiley \& Sons., 520 pp.

The Meteosat Archive User Handbook, 2001: Issue 2 (5), March. 